\title{
Industria 4.0, un Enfoque Discreto
}

\author{
Industry 4.0, a Discrete Approach
}

\author{
Jose Andrickson ${ }^{a}$, Edgar Chacón $^{b}$, María Pabón $^{c}$
}

Recibido: 8/9/2020 Aprobado: 27/10/2020

Cómo citar: Andrickson, J., Chacón, E., \& Pabón, M. (2020). Industria 4.0: un enfoque discreto. Ciencia, Ingenierias Y Aplicaciones, 3(2), 65-89. Doi: https://doi.org/10.22206/cyap.2020.v3i2.pp65-89

\section{Resumen}

En el diseño de la arquitectura de software y hardware, la re-configuración dinámica es un factor importante para la obtención de sistemas flexibles, robustos y escalables. Este proceso exige planificación, selección y el uso recurrente de métodos y recursos que permitan solventar los cambios externos e internos que se presentan en la producción. La investigación demuestra que los entornos dinámicos e integrados son enfoques para abordar las arquitecturas de sistemas modernos de producción. En este sentido, es necesario diseñar modelos matemáticos que incorporen la re-configuración dinámica con una filosofía holística como parte del mismo, aspectos en la actualidad considerados al momento del diseño de soluciones empresariales. Este trabajo considera un enfoque genérico basado en la filosofía de sistemas holónicos y técnicas modernas de sistemas ciberfísico, asi como el uso de los componentes de la Industria 4.0, como los gemelos digitales representado a través de sistemas a eventos discretos que permiten supervisar y gestionar el comportamiento deseado de un sistema de producción.

Palabras clave: industria; modelo de simulación; sistema experto; modelo matemático; sistemas de información.

\footnotetext{
a Instituto Especializado de Estudios Superiores Loyola, Facultad de Ingeniería, Dirección de Investigación, San Cristóbal, República Dominicana. Correo-e: jandrickson@ipl.edu.do b Universidad de Los Andes, Facultad de Ingeniera Laboratorio de Sistemas Discretos, Automatización e Integración, Mérida, Venezuela

c Univerlsidad Nacional Experimental del Táchira, Decanato de Investigación, Laboratorio de Instrumentación, Control y Automatización, Táchira, Venezuela
} 


\begin{abstract}
In the design of the software and hardware architecture, dynamic reconfguration is an important factor in obtaining flexible, robust and scalable systems. This process requires planning, selection and the recurrent use of methods and resources that allow solving the external and internal changes that occur in production. The research shows that dynamic and integrated environments are approaches to address the architectures of modern production systems, in this sense it is necessary to design mathematical models that incorporate dynamic re-configuration with a holistic philosophy as part of $i$, aspects currently considered when designing business solutions. This work considers a generic approach based on the philosophy of holonic systems and modern techniques of cyber-physical systems, as well as the use of the components of Industry 4.0, such as digital twins represented through systems at discrete events that allow monitoring and managing the desired behavior of a production system.
\end{abstract}

Keywords: Industry; simulation model; expert system; mathematical model; information systems. 


\section{Introducción}

La Industria 4.0 platea un diseño de la arquitectura de software y hardware de re-configuración dinámica para obtener sistemas flexibles, robustos y escalables. Este proceso exige planificación, selección y el uso recurrente de métodos y recursos para solventar los cambios externos e internos que se presentan en la producción.

La investigación demuestra que los entornos dinámicos e integrados son enfoques para abordar las arquitecturas de sistemas modernos de producción. En este sentido, es necesario diseñar lineamientos que incorporen la re-configuración dinámica (Tharumarajah, Wells, \& Nemes, 1998) y la filosofía holónica (Chacón, Besembel, Rivero, \& Cardillo, 2009), como parte del mismo; aspectos en la actualidad considerados al momento del diseńo de soluciones empresariales. Por tanto, este trabajo considera un enfoque genérico basado en la filosofía de sistemas holónicos y técnicas modernas de Sistemas Ciberfísicos (CPS), así como el uso de agentes.

Los Sistemas Ciberfísicos resultan de una integración de los sistemas computacionales (modelos y representaciones virtuales) y dispositivos inteligentes de proceso (Andrickson, Arellano, Caro, Pabón, \& Hernández, 2017); que permiten monitorear y tomar acciones en tiempo real de la producción, así como gestionar cualquier cambio necesario de manera preventiva o predictiva (Leitão, Karnouskos, \& Colombo, , 2016).

La dinámica e integración de los sistemas responden a una especificación de arquitectura de alto nivel, con manejo de información que permita evaluar el modelo del sistema a través del comportamiento en tiempo real de la imagen del mismo (modelo de referencia), desarrollando lo que se conoce como Gemelos Digitales, los cuales son implementados entre otras formas a través de modelos matemáticos, y se relacionan con el mundo real por medio de los sistemas ciberfísicos. Este tipo de arquitectura responde a una arquitectura dinámica, considerando Trentesaux (2009) existen tres tipos de arquitecturas: jerárquicas, heterárquicas y semi-heterárquicas; la arquitectura dinámica es una evolución de esta 
última, y la cual permite su autoconfiguración durante la ejecución del proceso (Jimenez et al., 2017).

Considerando los nuevos esquemas de automatización, fundamentados en sistemas virtuales y modelos matemáticos, la tendencia es tener nuevos sistemas automatizados que se fundamentan en las rápidas adaptaciones a los cambios externos e internos en el proceso (Andrickson, Chacón, Amaya, Pabón, \& Ramírez, 2012), lo cual flexibiliza el proceso productivo en tiempo real, y genera factores que promueven el desarrollo de una empresa productiva y dinámica adaptada a las exigencias del mercado global. Esta tendencia se orienta a la definición de la Industria 4.0 a través de sistemas autónomos y cooperantes (sistemas holónicos) con el uso de recursos modernos como el manejo de dispositivos y sistemas inteligentes (componentes de la Industria 4.0), además de la integración de gran cantidad de información (Big Data); lo cual redunda en una analítica de datos en tiempo real de la dinámica de la producción.

En la actualidad, los sistemas de manufactura responden de manera eficiente a las exigencias de mercado y a la demanda en tiempo real; dada su condición de adaptabilidad, re-configuración, y arquitectura dinámica (Jiménez et al., 2017), que junto a los sistemas de control y supervisión híbridos establecen cambios de comportamiento entre los diferentes niveles de automatización. En este mismo sentido, la comunicación y el manejo de la información se han convertido en elementos fundamentales para la definición de las empresas modernas y la Industria 4.0, donde el desarrollo de software ayuda al control, integración, monitoreo, diagnóstico y gestión de tareas o actividades dentro de la empresa (Karnouskos \& Leitão, 2017), a través de una modularidad descentralizada de los procesos.

\section{Descripción del sistema de producción}

\subsection{Metodología de implementación de Industria 4.0}

La Industria 4.0 se basa fundamentalmente en sistemas virtuales, autónomos, distribuidos, con factible desarrollo a través de los sistemas holónicos (Chacón et al, 2009), donde se considera un modelo producción de 
producto/proceso (Gamboa, Cardin, L'Anton, \& Castagna, 2015), que incluye el control, supervisión y gestión; siendo el sistema de información el punto focal del desarrollo e integración de la producción, lo que genera el incremento de la productividad de la empresa. Donde todos los procesos representan una dupla de información y recurso físico del proceso.

Un sistema es la interconexión entre varios componentes que cooperan entre sí con la finalidad de cumplir una meta de producción para la empresa. El propósito de la integración de los sistemas es trabajar de manera integrada como un solo elemento, donde se unen los componentes físicos y software y en los que el manejo del ciclo de vida y la informática constituye el enfoque moderno de optimización de la producción y de la Industria 4.0 (Bajer, 2008).

\subsection{Matemática para la Industria 4.0}

Existe una falsa creencia de que la Industria 4.0 es un conjunto de tecnologías de última generación conectadas al proceso productivo, pero nada más lejos de la realidad. De manera que hay que aclarar, que los aspectos fundamentales de la Industria 4.0 son: el manejo de gran cantidad de información, sistemas de toma de decisión con algoritmos matemáticos, y dispositivos inteligentes. Bajo esta premisa, la tecnología de la información emergente junto a los Sistemas Ciberfísicos, Big Data y el análisis de modelos de producción son los que definen: la interacción con clientes y proveedores, el manejo de la planificación y agenda de producción, y la exploración de datos de la empresa (Cherri, 2018).

Obtener la utilidad a la gran cantidad de datos recolectada de los diversos actores del proceso productivo, se logra por medio del uso de las matemáticas para la Industria 4.0, donde se aplican herramientas como: la minería de datos, inteligencia artificial, Machine Learning, Deep Machine, entre otras, lo cual permite a la empresa tomar decisiones de qué y cómo producir en función a un análisis de comportamiento de tendencias. La Industria 4.0 transforma los datos en decisiones, lo cual se traduce en la optimización de la operación de los procesos de la empresa y un mayor rendimiento de la productividad de la misma, en este sentido, las matemáticas y la ciencia computacional permiten el análisis en tiempo real y oportuno (Formaggia, 2017). 
Es importante asegurar un buen tratamiento de la información, para la construcción certera del conocimiento, a través del tratamiento de la información podemos establecer el punto de encuentro entre: la ciencia matemática, la ciencia computacional o informática, y la ingeniería de producción, que representa el conocimiento específico del proceso.

La utilización de los modelos matemáticos y la simulación, permiten complementar información parcial o no suficiente, para proveer un conocimiento completo del fenómeno en estudio y de esta manera tomar las decisiones adecuadas; por otra parte, el modelo matemático permite comparar la evolución de cualquier fenómeno o proceso, además de predecir su comportamiento a futuro. A través de la ciencia de la computación se busca desarrollar la integración del mundo real con el mundo virtual. Del trabajo de Formaggia (2017), se puede observar unión de los sistemas de cálculos con el mundo real para la toma de decisiones, definiendo la importancia de estas herramientas para la Industria 4.0.

\subsection{Administrative Shell}

En el entorno industrial y de ingeniería, la digitalización se ha convertido en el punto focal de los procesos productivos y la Industria 4.0. A medida que esta idea gana espacio, el concepto intuitivo de Administrative Shell (Capa de administración) se establece para definir el manejo de toda la información disponible de dispositivos y procesos. Por otra parte, la arquitectura de referencia para el modelo de Industria 4.0 (RAMI) define un esfuerzo global para la creación de empresas digitales o smart factories que se adaptan a los nuevos modelos, maximizando la capacidad de producción de las empresas (Grangel-González et al., 2016).

La Administrative Shell posee toda la información relativa a los activos o recursos físicos como máquinas y equipos, así como la información de producción, programación, planificación, configuración y operación relacionado a la lógica del proceso. En este sentido, también dentro del sistema se maneja información de manufactura y de complementación de funcionalidades y logística de negocio. La Administrative Shell es utilizada para almacenar información importante de los recursos del sistema, y cuya principal función es generar una red de manufac- 
tura con información de manejo se sistema, funciones, modelos, integración de recursos, y gestión de proceso productivo a través del bus virtual de automatización (Eclipse_Foundation, 2020), con modelos y sub-modelos de descripción establecido por la Asociación de Manufactura Eléctrica y Electrónica Alemana (ZVEI, 2018), definidos dentro de la clase recurso, que contienen información del ciclo de vida de producción (Maseu, Kolberg, \& Weyer, 2017), donde se establecen la condiciones específicas del objeto de la industria 4.0.

\section{Caso de aplicación}

Para el análisis del sistema y el modelo utilizaremos el enfoque de matemática discreta y su modelado con Sistemas a Eventos Discretos (DES), con lo cual se prueba la utilidad de la matemática en los procesos productivos, además de presentar la formalidad matemática en el desarrollo de autómatas.

El caso de estudio es el desarrollo de un control supervisorio del llenado de un tanque de abastecimiento de agua de un edificio (véase figura 1), el sistema consta de un depósito subterráneo que abastece al tanque aéreo y tres bombas de elevación de agua desde el depósito al tanque de abastecimiento. El control supervisorio es el encargado de mantener en el estado deseado (nivel y presión), basándose en flujo de entrada y salida, y condiciones de nivel del depósito subterráneo o cisterna. La integración de los componentes se logra a través de un URL como servidor de datos remoto. Con este ejercicio se presenta de manera sencilla la descripción de una empresa digitalizada con gestión y supervisión del proceso a través del manejo de información, que representa el gemelo digital del sistema real.

\subsection{Condiciones del sistema}

El proyecto consiste en modelar y supervisar el ciclo de vida del proceso a través del uso de matemática discreta, autómatas, y sistemas de información sobre el cual se define un gemelo digital. El sistema está constituido por: tres bombas de llenado, un compresor, una válvula de salida y tres válvulas de paso de agua en un solo sentido; adicionalmente, 
el sistema posee sensores de nivel tanto en el tanque de reserva o depósito como en el tanque de abastecimiento. Este último, posee un sensor de presión y sus condiciones de operación son las siguientes:

a) Las tres bombas no pueden estar encendidas al mismo tiempo.

b) La presión debe estar entre 80 y 100 libras.

c) A 95 libras de presión solo debe trabajar una bomba.

d) El nivel debe estar entre $40 \%$ y $60 \%$ del máximo del tanque.

e) Las bombas no deben succionar en vacío.

f) La válvula de salida debe cerrar mientras se llega a un nivel determinado.

En la figura 1 se muestra gráficamente la representación del sistema. Para el sistema y su accionamiento es necesario disponer de los permisos necesarios, para lo cual nos valemos de las herramientas computacionales y del uso de la información para generar la gestión con un repositorio de información que maneja las variables de permiso de ejecución del sistema, en el nivel de gestión del proceso.

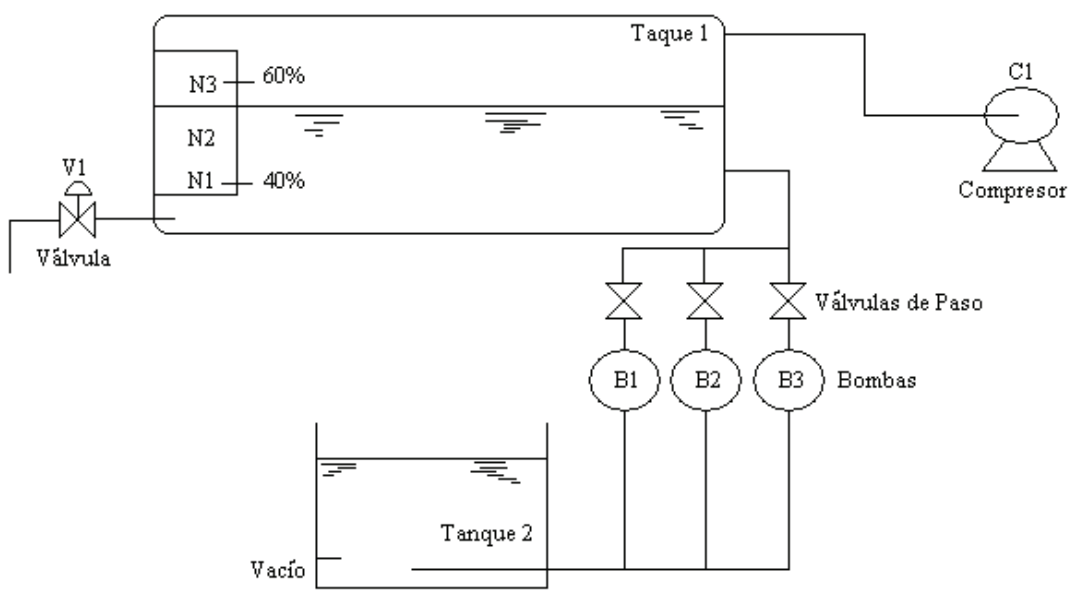

Figura 1. Sistema de abastecimiento de agua en un edificio

Cada elemento posee un Administation Shell que le permite representar sus estados y condiciones de operación, logrando la integración del sistema a través de la evolución de los autómatas y el sistema de supervisión. En la figura 2 se presenta la arquitectura en piso de planta. 


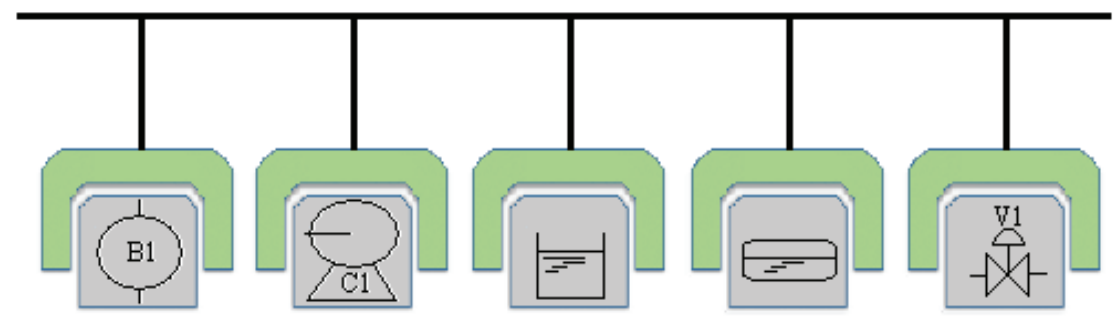

Figura 2. Arquitectura de Piso de Planta con Administration Shell

\subsubsection{Modelado del sistema}

Un proceso continuo puede proyectarse a una imagen discreta que es reflejo del estado de la región de operación en que se encuentra, y los eventos pueden ser generados por cambios en la región de operación, o por paradas y/o arranque de equipos, así como por cambios en la estructura dinámica del sistema continuo. La evolución de los sistemas continuos son los que determinan la ocurrencia de los eventos, que son capturados y determinados como estados del sistema; los mismos generan las acciones sobre para la evolución de estados y se describen por la transición de eventos discretos.

Para representar la conducta de los Sistemas a Eventos Discretos (DES), existen dos enfoques matemáticos: máquinas de estado finito y las redes de Petri. En el caso de estudio nos basamos en máquinas de estado finito y lenguajes formales de acuerdo con Cassandras y Lafortune (1999). Ya que se trabaja con un sistema híbrido de sistemas discretos basados en autómatas de estados finitos.

Se asume como condiciones iniciales que ambos tanques poseen condiciones similares de proceso, ambos llenos; donde el tanque de abastecimiento está cerrado, y la presión sobre el fluido es constante, controlada a través del compresor. Por lo tanto, para la representación matemática del sistema continuo, las variables definidas son las siguientes: 
$\mathrm{fi}=$ Flujo de entrada del valor de estado estables, $\mathrm{m}^{3} / \mathrm{seg}$

fo $=$ Flujo de salida del valor de estado estable, $\mathrm{m}^{3} / \mathrm{seg}$

$\mathrm{u}=$ Señal de entrada(s) de acuerdo al número de bomba(s) encendi$\mathrm{da}(\mathrm{s}),(0$ a 2$)$.

$\mathrm{A}=$ Área del tanque en estudio, $\mathrm{m}^{3}$

hi $=$ Nivel del tanque, $\mathrm{m}$

$\mathrm{c}=$ Condición del compresor, encendido o apagado $\left(\begin{array}{lll}0 & \mathrm{o} & 1\end{array}\right)$

$\mathrm{i}=$ Corresponde a cada tanque ( 1 o 2$)$.

La ecuación dinámica del sistema lineal y flujo laminar de cada tanque está dada por:

$$
A_{i} * \frac{b_{i}(t)}{t}=f_{i} * u-f_{o} *(1+c)
$$

\subsubsection{Modelado de control supervisorio a evento discreto}

El control supervisorio se desarrolla a través de autómatas de estado finito, con máquinas elementales de cada componente del sistema. Es de considerar que a medida que aumenta la complejidad de los sistemas, lo hace la representación de su conducta. Por tanto, los sistemas dinámicos híbridos pueden ser representados a través de sistemas a eventos discretos extrayendo su comportamiento de manera simplificada, donde la interacción entre componentes se define como la composición de sistemas a eventos discretos, como se presenta en la ecuación 2.

$$
\Phi^{c}\left(x_{0}, u\right)=\Phi_{K}\left(\Phi_{K-1}\left(\ldots \Phi_{2}\left(\Phi_{1}\left(x_{0}, t_{1}\right), t_{2}\right) \ldots, t_{k-1}\right), t_{k}\right)
$$

Los sistemas a eventos discretos se describen por máquinas de estados finitos y sus productos síncronos y asíncrono, generando la composición del autómata (Chacón, De Sarrazin, \& Khodr, 2001). En la figura 3, se muestra un esquema de un sistema con componentes interconectados que describen su modelo de interacción de información simplificado. 


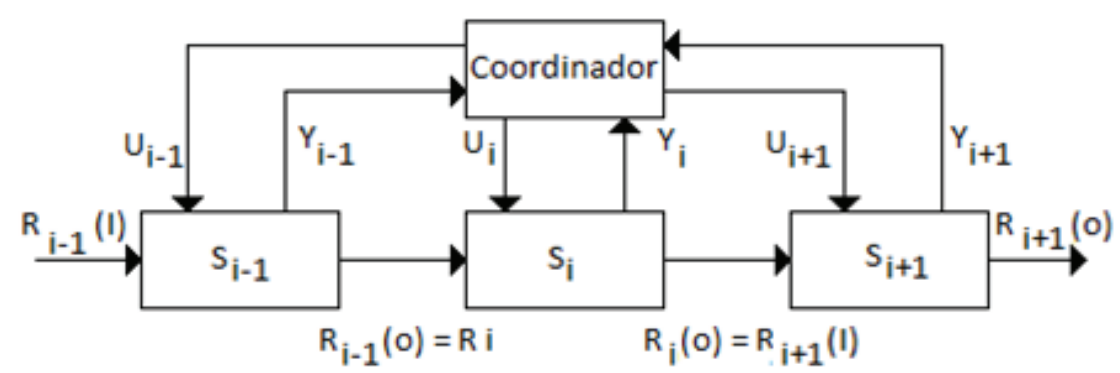

Figura 3. Sistemas con componentes interconectados

Para la integración de los elementos del sistema, se desarrolló el acoplamiento de las máquinas de estados finitos relacionando la información que fluye entre ellos para definir el modelo matemático que representará el gemelo digital del proceso; luego se definieron las condiciones de funcionamiento, estableciendo el lenguaje posible del sistema. Seguidamente, se establecieron las restricciones, de esta manera se reduce el número de posibles estados obtenidos a un lenguaje marcado o deseado del sistema. Los elementos tomados en cuenta y el número de estados y transiciones asociados derivados de los productos síncronos de los autómatas se muestran en la tabla 1 . De donde se observa que se tienen 576 estados como resultado de la interacción de los componentes del sistema.

Tabla 1. Elementos del sistema tomados para la sincronización

\begin{tabular}{lcc}
\hline Elemento & No de Estados & No de Transiciones \\
\hline Válvula 1 (V1) & 2 & 2 \\
\hline Bomba 1 (B1) & 2 & 2 \\
\hline Bomba 2 (B2) & 2 & 2 \\
\hline Bomba 3 (B3) & 2 & 2 \\
\hline Compresor 1 (C1) & 2 & 2 \\
\hline Nivel Tanque 1 (N1) & 3 & 4 \\
\hline Presión Tanque 1 (P1) & 3 & 4 \\
\hline Nivel Tanque 2 (T1) & 2 & 2 \\
\hline No de Estados & 576 & \\
Resultantes & & \\
\hline
\end{tabular}


La representación en diagrama de estados de cada componente se muestra en la figura 4, por ejemplo, para la válvula 1, el estado cero (0) corresponde a la condición de válvula cerrada y el estado uno (1) corresponde a la válvula abierta, las transiciones que le corresponde a la válvula son uno (1) para pasar de estado cerrado a abierto y tres (3) para pasar de estado abierto a cerrado. El mismo análisis se hace para cada componente del sistema, ver el resultado en la figura 4.
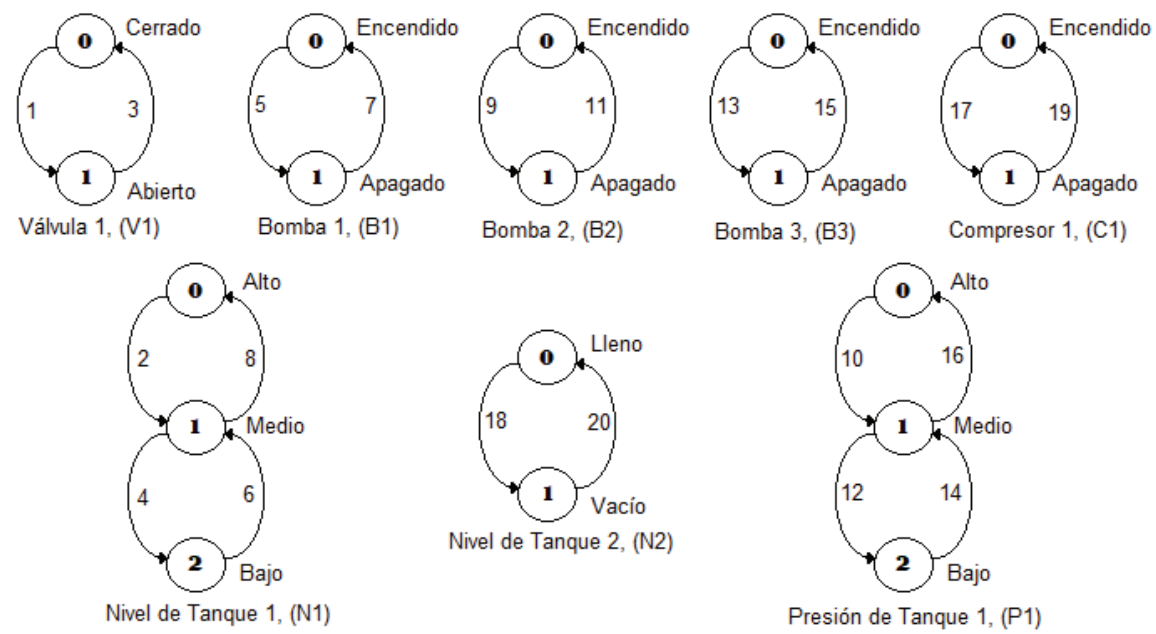

Figura 4. Representación de los elementos que componen el sistema de surtido de agua

Una vez definidas cada una de las máquinas elementales a trabajar, lo siguiente es acoplar cada elemento involucrado, utilizando como herramienta de cálculo el programa CTCT en la PC. El resultado:

- Estados: 576, lo cual coincide con los cálculos anteriores.

- Transiciones: 4992

El acoplamiento de estos elementos define el lenguaje físicamente posible del sistema. Las condiciones de funcionamiento de cada elemento se muestran en la figura 5. 


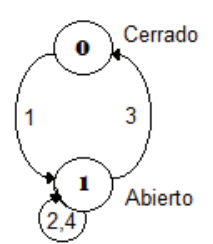

Condición 1, (R1)

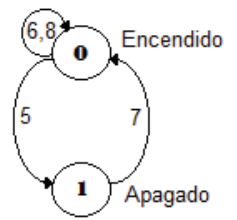

Condición 2, (R2)

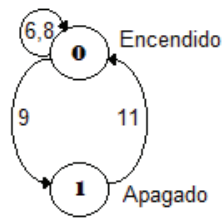

Condición 3, (R3)

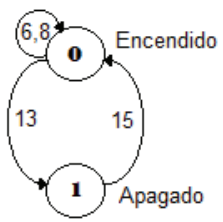

Condición 4, (R4) Condición 5, (R5)

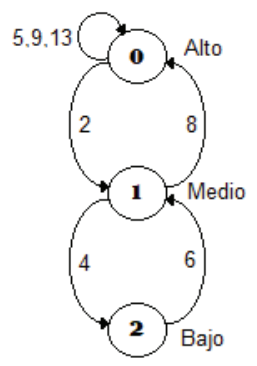

Condición 6, (R6)

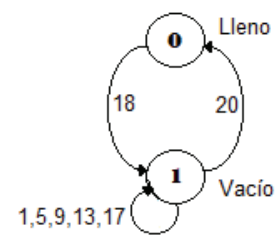

Condición 8, (R8)

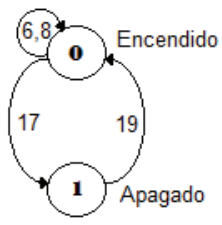

Figura 5. Condiciones para el Autómata del Sistema

\subsubsection{Definición del autómata}

Para el desarrollo del modelo de implementación simplificado, se decidió, por inspección y obviando algunos estados de baja probabilidad, establecer las condiciones deseadas como operador, lo cual resulta en un subconjunto del lenguaje generado, lo que genera una condición particular de funcionamiento del sistema que cumple las funciones deseadas, considerando de manera consciente que se están obviando algunos estados y transiciones del sistema. De esta manera se define el sistema con los estados marcados, incluyendo algunas restricciones para obtener el sistema deseado. El lenguaje marcado del proceso, es un subconjunto del lenguaje físicamente posible y se representa en la tabla 2, donde el estado $\mathrm{X}$ es activo y el estado $\underline{\mathrm{X}}$ es inactivo, con $\mathrm{X}$ representado en cualquier elemento del sistema (V, C, B1, B2, B3). 
Tabla 2. Lenguaje marcado para la definición del autómata

\begin{tabular}{|c|c|c|}
\hline $\begin{array}{l}\text { Condición de } \\
\text { Elementos }\end{array}$ & Condiciones & Leyenda \\
\hline$\underline{\mathrm{V}} \underline{\mathrm{C}} \underline{\mathrm{B} 1} \underline{\mathrm{B} 2} \underline{\mathrm{B} 3}$ & N1 P3 T2 & N3 = Nivel Alto \\
\hline$\underline{\mathrm{V}} \underline{\mathrm{C}} \mathrm{B} 1 \underline{\mathrm{B} 2} \underline{\mathrm{B} 3}$ & N1 P3 T1 & N2 $=$ Nivel Medio \\
\hline$\underline{\mathrm{V}} \underline{\mathrm{C}} \underline{\mathrm{B} 1} \mathrm{~B} 2 \underline{\mathrm{B} 3}$ & N1 P3 T1 & N1 = Nivel Bajo \\
\hline$\underline{\mathrm{V}} \underline{\mathrm{C}} \underline{\mathrm{B} 1} \underline{\mathrm{B} 2} \mathrm{~B} 3$ & N1 P3 T1 & P3 = Presión Alta \\
\hline$\underline{\mathrm{V}} \underline{\mathrm{C}} \mathrm{B} 1 \mathrm{~B} 2 \underline{\mathrm{B} 3}$ & N1 (P1 y P3) T1 (No procedente) & P1 = Presión Baja \\
\hline$\underline{\mathrm{V}} \underline{\mathrm{C}} \mathrm{B} 1 \underline{\mathrm{B} 2} \mathrm{~B} 3$ & N1 (P1 y P3) T1 (No procedente) & T1 = Depósito Lleno \\
\hline$\underline{\mathrm{V}} \underline{\mathrm{C}} \underline{\mathrm{B} 1} \mathrm{~B} 2 \mathrm{~B} 3$ & N1 (P1 y P3) T1 (No procedente) & T2 = Depósito Vacío \\
\hline$\underline{\mathrm{V}} \mathrm{C} \underline{\mathrm{B} 1} \underline{\mathrm{B} 2} \underline{\mathrm{B} 3}$ & N1 P1 T2 & $\mathrm{V}=$ Válvula Abierta \\
\hline$\underline{\mathrm{V}} \mathrm{C} B 1 \underline{\mathrm{B} 2} \underline{\mathrm{B} 3}$ & N1 (P1 y P3) T1 (No procedente) & $\underline{\mathrm{V}}=$ Válvula Cerrada \\
\hline$\underline{\mathrm{V}} \mathrm{C} \underline{\mathrm{B} 1} \mathrm{~B} 2 \underline{\mathrm{B} 3}$ & N1 (P1 y P3) T1 (No procedente) & $\mathrm{C}=$ Compresor Encendido \\
\hline$\underline{\mathrm{V} C} \underline{\mathrm{B} 1} \underline{\mathrm{B} 2} \mathrm{~B} 3$ & N1 (P1 y P3) T1 (No procedente) & $\underline{\mathrm{C}}=$ Compresor Apagado \\
\hline$\underline{\mathrm{V}} \mathrm{C} \mathrm{B} 1 \mathrm{~B} 2 \underline{\mathrm{B} 3}$ & N1 P1 T1 & B1 = Bomba 1 Encendida \\
\hline$\underline{\mathrm{V}} \mathrm{C}$ B1 $\underline{\mathrm{B} 2} \mathrm{~B} 3$ & N1 P1 T1 & $\underline{B 1}=$ Bomba 1 Apagada \\
\hline$\underline{\mathrm{V}} \mathrm{C} \underline{\mathrm{B} 1} \mathrm{~B} 2 \mathrm{~B} 3$ & N1 P1 T1 & $\mathrm{B} 2=$ Bomba 2 Encendida \\
\hline $\mathrm{V} \underline{\mathrm{C}} \underline{\mathrm{B} 1} \underline{\mathrm{B} 2} \underline{\mathrm{B} 3}$ & $(\mathrm{~N} 2$ o N1) P3 T2 & $\underline{\mathrm{B} 2}=$ Bomba 2 Apagada \\
\hline $\mathrm{V} \underline{\mathrm{C}} \mathrm{B} 1 \underline{\mathrm{B} 2} \underline{\mathrm{B} 3}$ & (N2 o N1) P3 T1 & B3 = Bomba 3 Encendida \\
\hline $\mathrm{V} \underline{\mathrm{C}} \underline{\mathrm{B} 1} \mathrm{~B} 2 \underline{\mathrm{B} 3}$ & (N2 o N3) P3 T1 & $\underline{\mathrm{B} 3}=$ Bomba 3 Apagada \\
\hline $\mathrm{V} \underline{\mathrm{C}} \underline{\mathrm{B} 1} \underline{\mathrm{B} 2} \mathrm{~B} 3$ & (N2 o N3) P3 & \\
\hline $\mathrm{V} \underline{\mathrm{C}} \mathrm{B} 1 \mathrm{~B} 2 \underline{\mathrm{B} 3}$ & $\begin{array}{c}(\mathrm{N} 2 \text { o N3) }(\mathrm{P} 3 \text { y } \mathrm{P} 1) \mathrm{T} 1 \text { (No } \\
\text { procedente) }\end{array}$ & \\
\hline $\mathrm{V} \underline{\mathrm{C}} \mathrm{B} 1 \underline{\mathrm{B} 2} \mathrm{~B} 3$ & $\begin{array}{c}(\mathrm{N} 2 \text { o N3) }(\mathrm{P} 3 \text { y } \mathrm{P} 1) \mathrm{T} 1 \text { (No } \\
\text { procedente) }\end{array}$ & \\
\hline $\mathrm{V} \underline{\mathrm{C}} \underline{\mathrm{B} 1} \mathrm{~B} 2 \mathrm{~B} 3$ & $\begin{array}{c}(\mathrm{N} 2 \text { o N3) }(\mathrm{P} 3 \text { y } \mathrm{P} 1) \mathrm{T} 1 \text { (No } \\
\text { procedente) }\end{array}$ & \\
\hline $\mathrm{VCC} \underline{\mathrm{B} 1} \underline{\mathrm{B} 2} \underline{\mathrm{B} 3}$ & (N2 o N3) P1 T2 & \\
\hline $\mathrm{VC} B 1 \underline{\mathrm{B} 2} \underline{\mathrm{B} 3}$ & $\begin{array}{c}(\mathrm{N} 2 \text { o N3) }(\mathrm{P} 3 \text { y } \mathrm{P} 1) \mathrm{T} 1 \text { (No } \\
\text { procedente) }\end{array}$ & \\
\hline $\mathrm{VCC} \underline{\mathrm{B} 1} \mathrm{~B} 2 \underline{\mathrm{B} 3}$ & $\begin{array}{c}(\mathrm{N} 2 \text { o N3) }(\mathrm{P} 3 \text { y } \mathrm{P} 1) \mathrm{T} 1(\mathrm{No} \\
\text { procedente })\end{array}$ & \\
\hline $\mathrm{VCC} \underline{\mathrm{B} 1} \underline{\mathrm{B} 2} \mathrm{~B} 3$ & $\begin{array}{c}(\mathrm{N} 2 \text { o N3) }(\mathrm{P} 3 \text { y } \mathrm{P} 1) \mathrm{T} 1 \text { (No } \\
\text { procedente) }\end{array}$ & \\
\hline V C B1 B2 $\underline{\mathrm{B} 3}$ & (N2 o N3) P1 & \\
\hline $\mathrm{VC} \mathrm{B1} \underline{\mathrm{B} 2} \mathrm{~B} 3$ & $(\mathrm{~N} 2$ o N3) P1 & \\
\hline $\mathrm{V} \mathrm{C} \underline{\mathrm{B} 1} \mathrm{~B} 2 \mathrm{~B} 3$ & $(\mathrm{~N} 2$ o N3) P1 & \\
\hline
\end{tabular}


Esta representación es similar a una programación de un autómata programable, considerando que es posible que se presenten estados no tomados en cuenta. En caso contrario, implicaría mayor detalle en el modelo del sistema (gemelo digital), por lo cual se debe hacer una descripción de alerta de los estados no deseados, es importante considerar que la representación del proceso físico del sistema en un autómata programable describe un Sistemas Ciberfísico. Considerando lo expuesto, el accionamiento de elementos del sistema debe ser:

- Condición de bombas: solo debe estar activa una bomba con presión alta.

- Condición de válvula: solo se debe cerrar cuando el nivel este bajo.

- Condición de compresor: solo debe ser encendido con presión baja.

La figura 6 muestra el autómata con estados marcados sin reducción de datos por verificación.

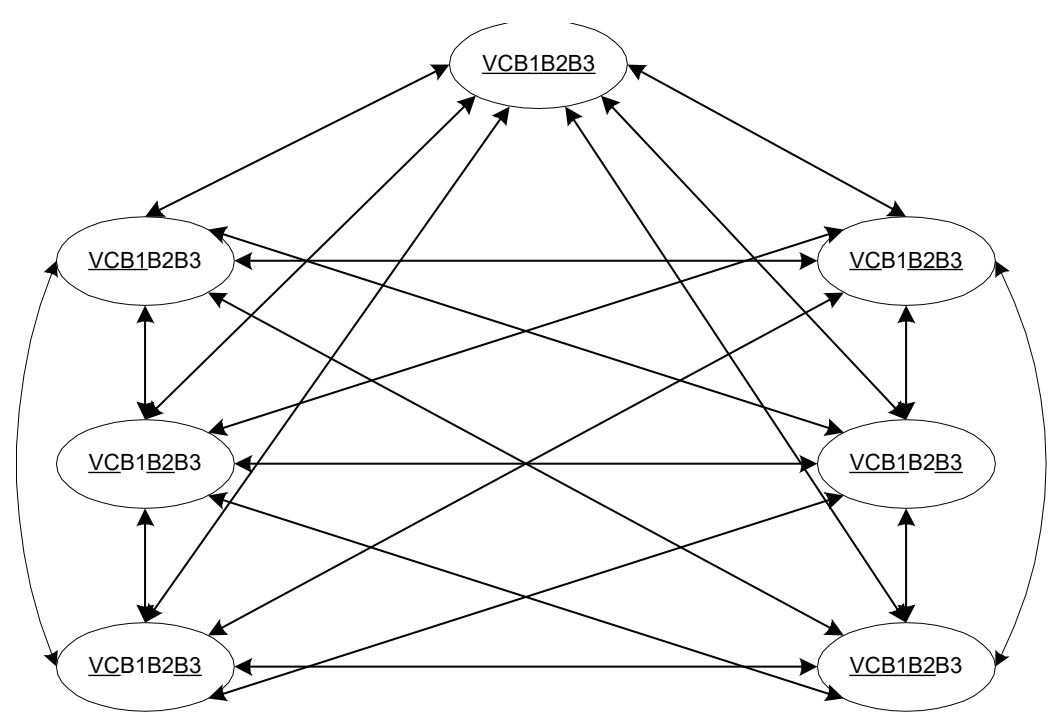

Figura 6. Autómata sin restricciones

En la figura 7, se observa la reducción de los estados de la figura 6, los cuales fueron obtenidos por inspección de la tabla 2 , donde se nota una reducción del lenguaje físico del sistema, obteniendo los estados reducidos del sistema y el autómata mínimo. 


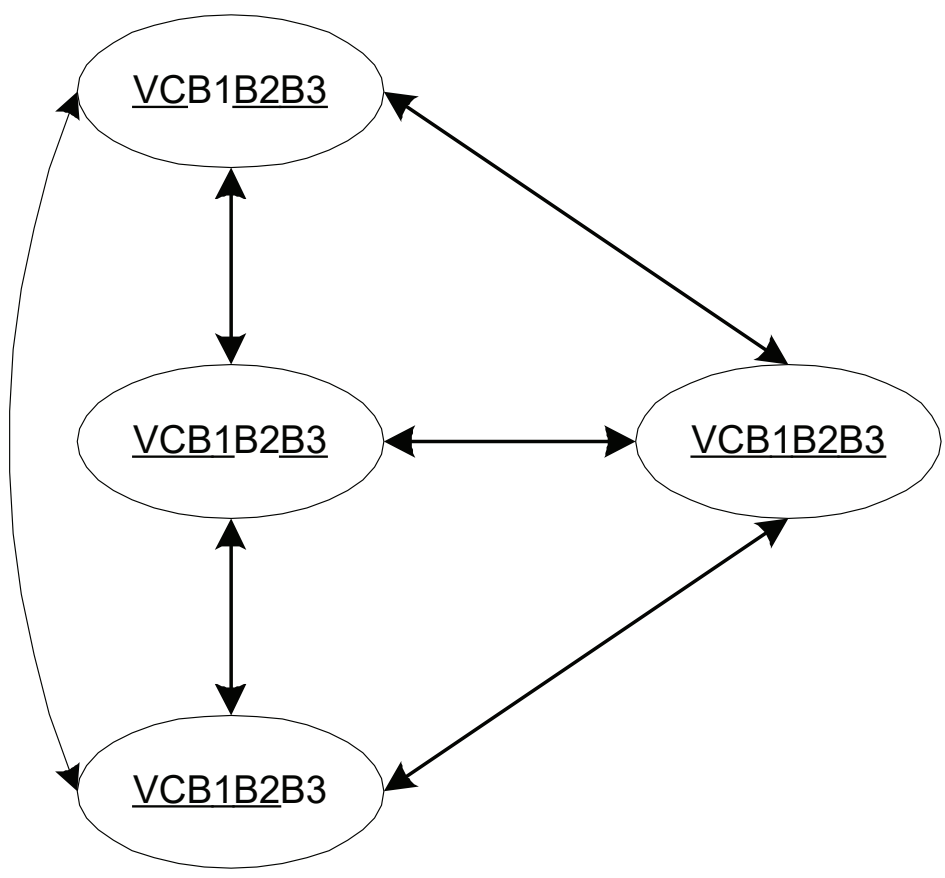

Figura 7. Autómata con restricciones

Como resultado de la implementación del autómata reducido del sistema se obtiene el lenguaje auto-controlado en los autómatas, donde se describe un proceso depurado que permite mejorar la trayectoria y comportamiento, como un gemelo digital, permitiendo supervisar y controlar el proceso real, representando un sistema de manufactura inteligente basado en la Industria 4.0 de un sistema de control de surtir agua en un edificio; lo cual resulta en un ejemplo elemental, pero con análisis completo de su comportamiento y representación de estudio matemático y su gestión de información.

Es importante considerar que el sistema representa un proceso de Industria 4.0 por lo cual posee una interacción de gestión de proceso que va en el análisis de la información de los Sistemas Ciberfisicos y lo gemelos digitales. Considerando los autómatas como la representación matemática y la descripción del modelo (gemelo digital) implantado como sistemas Ciberfísico con autómata programable (PLC). En la figura 8, se presenta el esquema de automatización de implementación. 


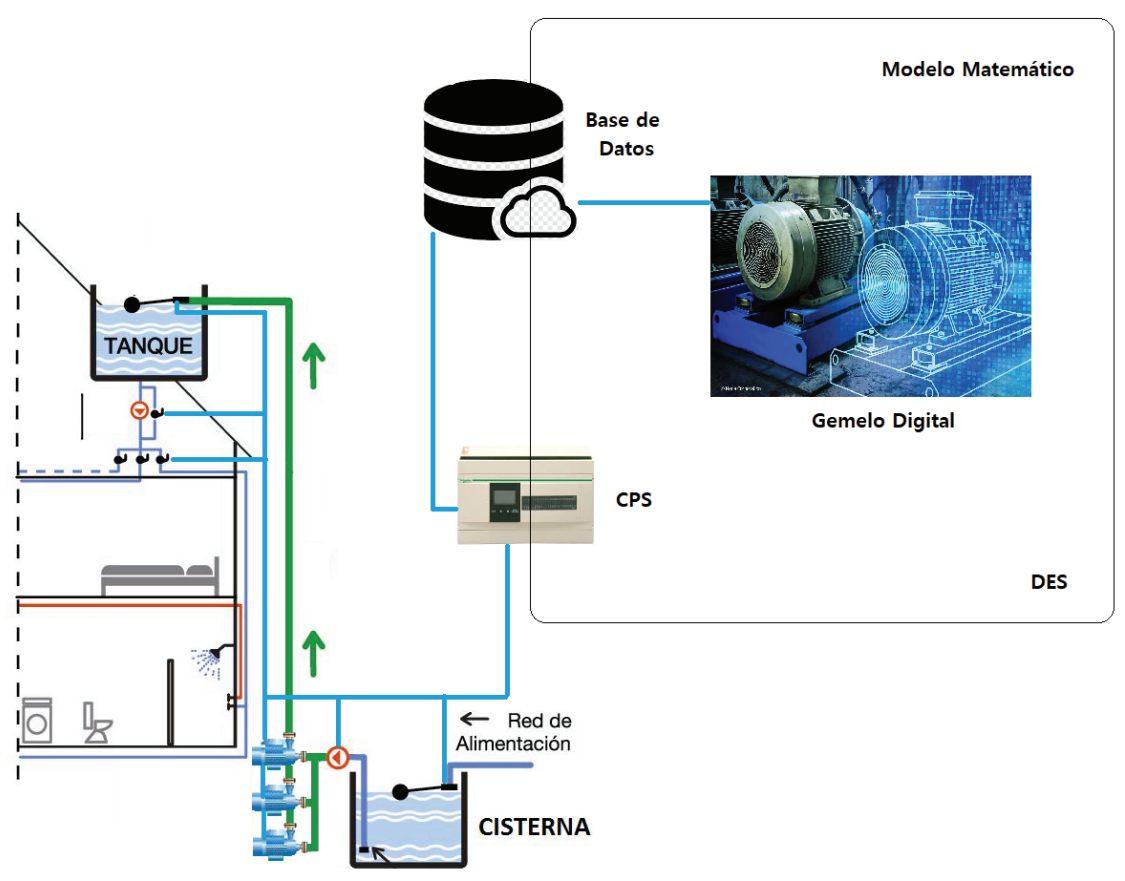

Figura 8. Modelo del Sistemas de Control, Supervisión y Gestión de Tanque Surtidor

\section{Resultados}

Los modelos matemáticos y la Industria 4.0 permiten la integración de los componentes que forman parte de un sistema productivo, para ello se hace uso de los avances de la comunicación, la información, la electrónica y la computación.

La integración de los sistemas heterogéneos, trae consigo grandes beneficios como la reducción de costos, aumento de flexibilidad en los procesos y reducción de fallas en los sistemas. Otro aspecto importante dentro de la integración es la capacidad de interoperación de diferentes componentes (Chacón, 2003).

En este artículo, además de analizar y desarrollar un sistema supervisor para un tanque de abastecimiento de agua de un edificio, también 
se hace el uso de la nueva tendencia de la automatización en el aspecto de la integración de los componentes, donde se desarrolla un control en forma remota de las variables como presión y nivel del tanque de abastecimiento y del tanque de depósito, a través de comunicación TCP/IP.

Cuando se busca integrar varios equipos o sistemas, lo primero es plantearse el medio por el cual se van a comunicar y el lenguaje que van a conversar entre sí. Para ello se necesita que todos los equipos que componen el sistema sean capaces de comunicarse en el medio o tener un intérprete que le permita esta comunicación. Seguidamente se deben desarrollar los modelos matemáticos representativos para hacer un estudio específico de interacción del sistema y la gestión de información entre los componentes, de aquí se plantea la arquitectura de automatización.

Uno de los aspectos para la integración de los sistemas es la capacidad de digitalización de los procesos y componentes, entendiendo su entorno y comunicación como sistemas pares entre ellos y con otros niveles del sistema.

El modelado matemático y la integración de los diferentes sistemas se hace con el uso de subrutinas creadas en $\mathrm{Matlab}^{\oplus}$, que además de modelar el proceso son capaces de interactuar con las variables desde un servidor en internet y en función de los valores de estas, ejecutar tareas determinadas. Para ver el funcionamiento del proceso y de cómo se accionan las válvulas, se realizó una simulación en el programa Stateflow de Matlab ${ }^{\oplus}$. Del cual se muestran las figuras 9 y 10. Esto establece el comportamiento del sistema representado como el Gemelo Digital del proceso real. Esta virtualización provoca cambios, lo cual promueve un sistema de producción flexible (DLG, 2019).

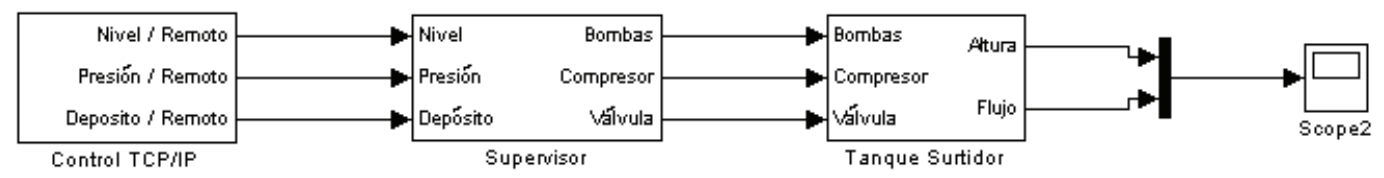

Figura 9. Simulación en $\mathrm{Matlab}^{\oplus}$ 
También se tiene el control a través de una Edge Computing (TCP/ IP), que es un llamado a funciones básicas de $\mathrm{Matlab}^{\circledR}$, las cuales, combinadas entre sí, establecen una comunicación con archivos en la base de datos, los cuales poseen las condiciones de nivel y presión que están sensando los diferentes componentes del sistema y que serán enviados al supervisor.

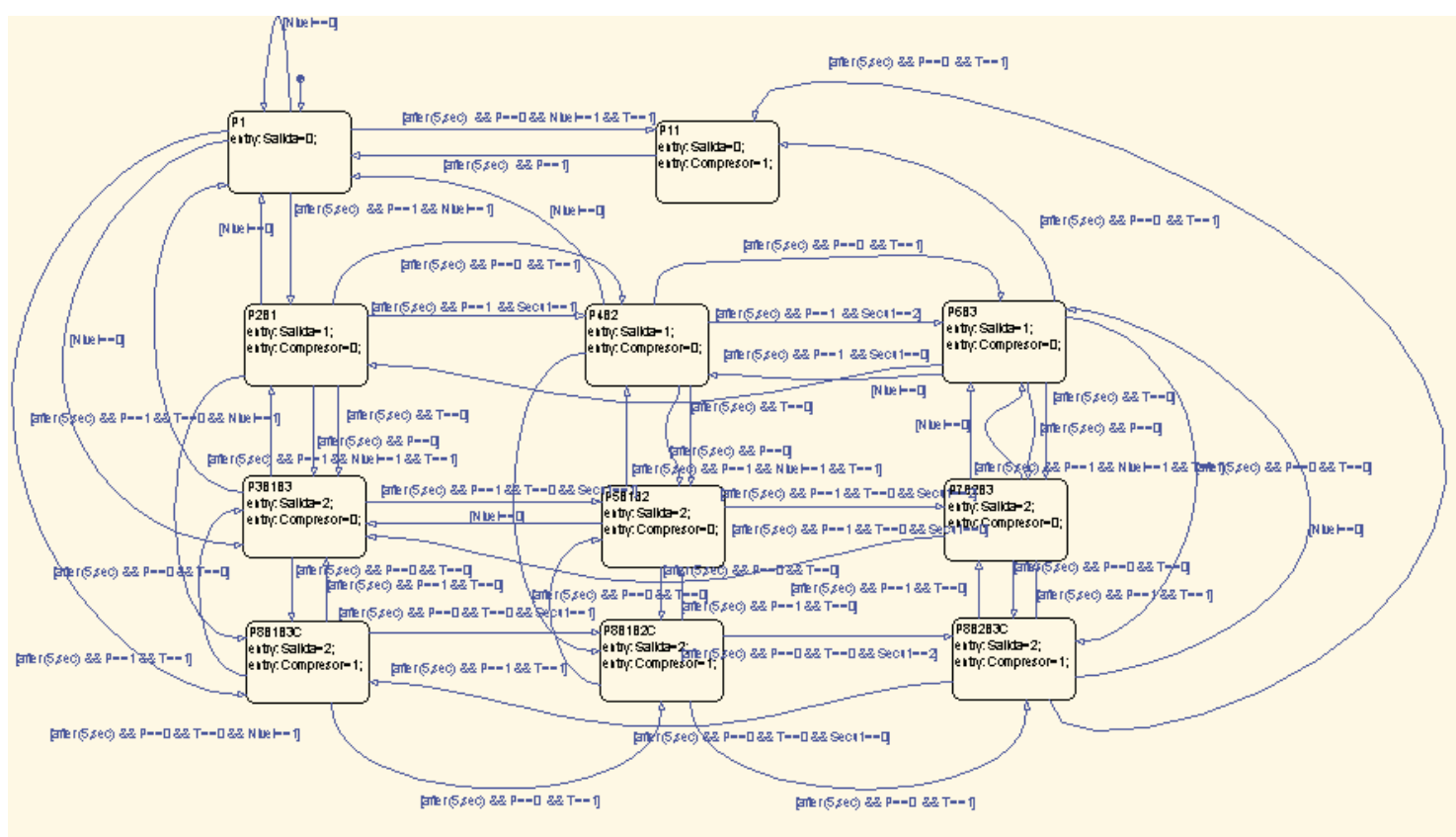

Figura 10. Secuencia del supervisor a eventos discretos

En la figura 10, se muestra el diseño del supervisor, el cual se desarrolló a través de eventos de estados como: una bomba encendida de forma rotativa, dos bombas encendidas de forma rotativa, el compresor encendido o apagado y combinaciones de estos. Las transiciones entre los estados se dan con condiciones como depósito: vacío o lleno; nivel del tanque: alto o bajo; presión en el tanque: alta o baja; tiempo de encendido de las bombas. La simulación del mismo se implementó en la herramienta Stateflow de Matlab ${ }^{\oplus}$. 
Para la simulación del sistema, se presentó la siguiente secuencia de condiciones:

1. La condición de inicio: presión está en alto y el nivel del tanque de depósito se encuentra en alto, además, el nivel del tanque surtido se encuentra en estado de alto.

2. A los 50 minutos, el nivel del tanque de abastecimiento se encuentra en condición de bajo.

3. A los 100 minutos, el sistema entra en condición de presión baja y a los 150 se recupera la presión y entra en condición alta o normal.

4. A los 200 minutos, el nivel se recupera y entra en condición de alto.

5. A los 250 minutos el tanque de depósito reporta condición de vacío, la cual se recupera a los 300 minutos.

El comportamiento del sistema es el adecuado como se aprecia en la figura 11 .
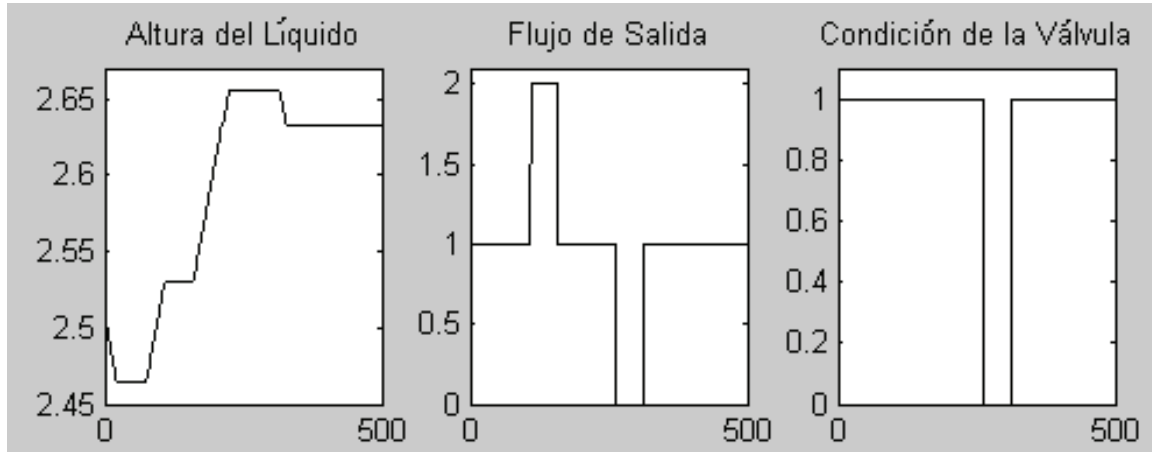

\section{Bombas Encendidas}

Condición del Compresor
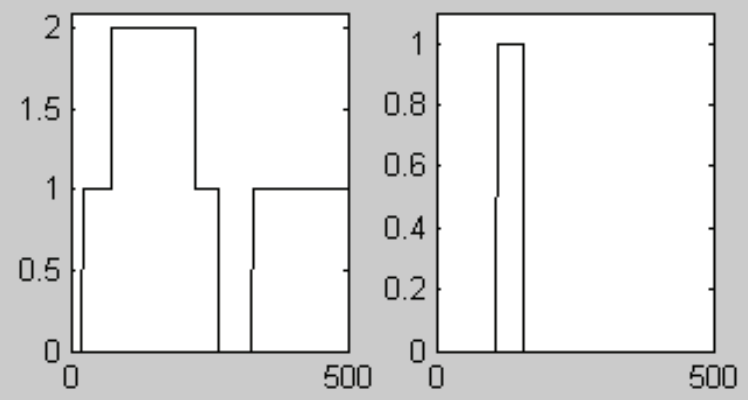

Nivel del Depósito

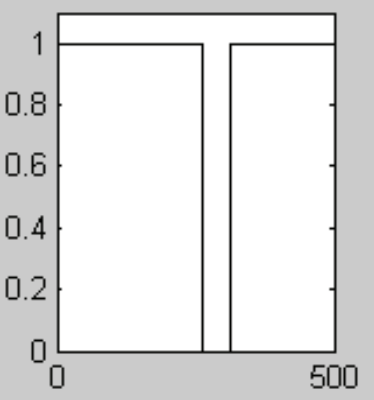

Figura 11. Respuesta del sistema de tanque de abastecimiento de agua 
$\mathrm{Al}$ arrancar el sistema, el tanque se encuentra en $2,5 \mathrm{~m}$, la cual es una condición de inicio preestablecida que establece la estabilización del sistema, con flujo de salida constante y solo una bomba encendida, que se alterna entre las tres disponibles. Cuando el nivel del tanque de abastecimiento se encuentra en estado bajo, se encienden dos de las tres bombas que funcionan de forma rotativa para restablecer el nivel adecuado.

En la modelación se puede apreciar que en el minuto 100 el sistema reporta una condición de presión baja, que se compensa con el encendido del compresor y dos bombas, por lo cual el nivel se compensa, ya que el de flujo de entrada aumenta y estabiliza el nivel del tanque de abastecimiento. A los 150 minutos, el sistema entra en condición de presión normal, y el nivel del tanque de abastecimiento sigue incrementándose, hasta que llega a estado de alto y comienzan a trabajar en rotación de una sola bomba, produciendo que el nivel en el tanque se nivela en un valor deseado.

Entre los minutos 200 y 300 el depósito cisterna indica nivel de depósito bajo, ocasionando un cierra de la válvula de salida, se apagan las bombas y se hace cero el flujo de salida, donde se cumple con las condiciones establecidas. Una vez vuelve a llenar el tanque cisterna, el flujo de salida vuelve a ser constante al igual que el de entrada, y en este sentido, el nivel del tanque se estabiliza e inicia el funcionamiento normal del sistema.

De estos resultados, se puede observar que la simulación del sistema real, reflejada a través de un Gemelo Digital descrito por medio de autómatas con sistemas a eventos discretos, crean un sistema flexibilidad, tolerancia a fallas y un uso eficiente de los recursos, lo cual promueve un sistema dinámico dentro de la empresa que le permite adaptarse a las exigencias globales, orientando las empresas al nuevo paradigma de Industria 4.0. Además, es de notar que al simular las condiciones de nivel y presión de forma remota permite desarrollar una simulación de de integración de múltiples niveles de automatización. 


\section{Conclusiones}

El artículo muestra la propuesta de implementación de los parámetros y definición de Industria 4.0 en un caso de uso cotidiano, que sirve para determinar la gran importancia de las herramientas matemáticas y simulación para representar el nuevo esquema de industria digital y sus grandes virtudes para el control de los procesos sin necesidad de implementar grandes algoritmos matemáticos de ecuaciones complejas en tiempo continuo, ya que la solución se da a través de la matemática discreta y los autómatas.

Además, se representa la integración del sistema a través del uso de la tecnología de la información y los repositorios de datos, por medio del cual se establece el nivel de gestión del proceso productivo, ya que para ejecutarse algunas acciones es necesario un permisivo informático de que esté disponible para esta actividad.

Por otra parte, presenta el modelado del sistema hidroneumático a través de una ecuación diferencial, luego se estudió a grandes rasgos el comportamiento del lenguaje que define la planta y las condiciones del sistema. El mismo se implementa haciendo uso de la herramienta Stateflow de Matlab ${ }^{\bullet}$. Luego se desarrolló el sistema de gestión de información a través de un repositorio de datos, donde se encuentran los permisos de ejecución de las acciones de supervisión del sistema.

Se puede establecer que se presentó la implementación de la Industria 4.0 con sus componentes desarrollados a través de un sistema supervisor y de gestión con el uso de un gemelo digital desarrollado por sistema a evento discreto y autómatas de estado finitos. De donde se observa las bondades de los sistemas inteligentes en toma de decisiones e integración de los sistemas.

\section{Agradecimientos}

Esta investigación se da como resultado de la colaboración de la Universidad de Los Andes (ULA) y Universidad Nacional Experimental del Táchira (UNET), Venezuela, en el área de automatización, en 
conjunto con el Instituto Especializado de Estudios Superiores Loyola (IEESL), con la finalidad de establecer lazos académicos que permitan un crecimiento en el área de investigación y aplicación del conocimiento de la Industria 4.0 al entorno dominicano.

El trabajo no recibió financiamiento, ya que es producto de una investigación colaborativa entre los autores.

\section{Referencias}

Andrickson, J., Arellano, M., Caro, A., Pabon, M., \& Hernandez, C. (2017). Sistema de Informacion en Control de Proceso. Revista Cientifica UNET. 29(2), 74-90.

Andrickson, J., Chacón, E., Amaya, J., Pabón, M., \& Ramirez, A. (2012). Arquitectura de Automatización Basada en Holón Industrial . Revista Cientifica UNET. 24(1), 25-36.

Bajer, M. (2008). Control Systems Integration using OPC Standard. ResearchGate, 1-115.

Cassandras, C., \& Lafortune, S. (1999). Introduction to Discrete Event Systems. Boston: Boston University.

Chacón, E., Besembel, I., \& Hennet, J. (2004). Coordination and Optimization in Oil \& Gas Production Complexes. Computers in Industry. 53(1), 17-37.

Chacón, E., Besembel, I., Rivero, D., \& Cardillo, J. (2009). Embedded holonics systems in production process: holonic unit of production. Revista Técnica de la Facultad de Ingeniería Universidad del Zulia, 32(1), 3-13.

Chacón, E., De Sarrazin, G., \& Khodr, Y. (2001). Coupled Dynamic for Industrial Complex Systems. ISA Transactions. 47(3), 305-319.

Cherri, L. (2018). The Mathematics of Industry 4.0. [entrada en blog]. Recuperado de https://medium.com/@luizcherri/the-mathematics-of-industry-4-0-81904d7bd3cb

Corporacion Dominicana de Empresas Eléctricas Estatales (CDEEE). (2012). Experto estima red eléctrica inteligente ayudará solucionar problemas eléctricos del país. Santiago - República Dominicana: Corporacion Dominicana de Empresas Eléctricas Estatales. 
DLG. (15 de diciembre de 2019). Industry 4.0 - Summ ary report. Recuperado de https://www.cenit.com/fileadmin/dam/Corporate/PDFs/2015_5_Expertenwissen_E.pdf Eclipse_Foundation. (16 de marzo de 2020). Eclipse BaSyx: A Middleware for Industry 4.0: Recuperado de https://www.eclipse.org/community/eclipse_ newsletter/2019/july/basyx.php.

Formaggia, L. (2017). Mathematics and Industry 4.0. International CAE Conference.

Gamboa, F., Cardin, O., L'Anton, A., \& Castagna, P. (2015). Implementation of a Process Orchestration Model in a Service Oriented Holonic Manufacturing System. Elsevier, ScienceDirect. IFAC Paper Online, 48(3), 1111-1116.

Grangel-González, I., Halilaj, L., Coskun, G., Auer, S., Collarana, D., \& Hoffmeister, M. (2016). Towards a Semantic Administrative Shell for Industry 4.0 Components. IEEE Tenth International Conference on Semantic Computing (ICSC). California, USA, 1-14.

Jimenez, J. F. et al. (2017). Dynamic and hybrid architecture for the optimal reconfiguration of control systems: Application to manufature control. (PhD diss). Universite de Valenciennes et du Hainaut-Cambresis. Edinburgo.

Karnouskos, S., \& Leitão, P. (2017). Key Contributing Factors to the Acceptance of Agents in Industrial Environments. IEEE Transactions on Industrial Informatics, 696-703.

Leitão, P., Colombo, A., \& Karnouskos, S. (2015). Industrial automation based on cyber-physical systems technologies: Prototype implementations and challenges. Computers in Industry, 1-25.

Maseu, E., Kolberg, D., \& Weyer, S. (2017). Exemplary transfer of the RAMI 4.0 Administration Shell to the SmartFactoryKL System Architecture for Industrie 4.0 Production Systems. Whitepaper, Technologie-Initiative SmartFactory KL, Kaiserslautern. Recuperado de https://smartfactory.de/wp-content/uploads/2017/11/SF_WhitePaper_2-1_EN-1.pdf

Tharumarajah, A., Wells, A., \& Nemes, L. (1998). Comparison of emerging manufacturing concepts," SMC'98 Conference Proceedings. IEEE International Conference on Systems, Man, and Cybernetics (Cat. No.98CH36218), San Diego, CA, USA, 325 - 331. 
Trentesaux, D. (2009). Distributed control of production systems. Engineering Applications of Artificial Intelligence, 22(7), 971 - 978.

ZVEI. (7 de abril de 2017). Examples of the Asset Administration Shell for Industrie 4.0 Components - Basic Part Recuperado de https:// www.zvei.org/fileadmin/user_upload/Presse_und_Medien/Publikationen/2017/April/Asset_Administration_Shell/ZVEI_WP_ Verwaltungschale_Englisch_Download_03.04.17.pdf 